\title{
The uniqueness of solution for initial value problems for fractional differential equation involving the Caputo- \\ Fabrizio derivative
} Check for updates

\author{
Shuqin Zhang ${ }^{\mathrm{a}, *}$, Lei Hu ${ }^{\mathrm{b}}$, Sujing Sun ${ }^{\mathrm{c}}$ \\ ${ }^{a}$ China University of Mining and Technology (Beijing), School of Science, Beijing 100083, P. R. China. \\ ${ }^{b}$ Shandong Jiaotong University, School of Science, Jinan 250357, Shandong, P. R. China. \\ ' Shandong University of Science and Technology, College of Mathematics and System Science, Qingdao 266590, Shandong, P. R. \\ China.
}

Communicated by D. Baleanu

\begin{abstract}
In this paper, we study some results about the expression of solutions to some linear differential equations for the CaputoFabrizio fractional derivative. Furthermore, by the Banach contraction principle, the unique existence of the solution to an initial value problem for nonlinear differential equation involving the Caputo-Fabrizio fractional derivative is obtained.
\end{abstract}

Keywords: The Caputo-Fabrizio fractional derivative, initial value problem, fractional differential equations, Banach contraction principle, uniqueness.

2010 MSC: 26A33, 34B15.

(C)2018 All rights reserved.

\section{Introduction}

In this paper, we consider the following initial value problems for fractional differential equation

$$
\left\{\begin{array}{l}
{ }^{C F} D^{\alpha} x(t)=g\left(t, x,{ }^{C F} D^{\beta} x\right)-g\left(0, x(0),{ }^{C F} D^{\beta} x(0)\right), 0<t<+\infty \\
x(0)=x_{0} \in R
\end{array}\right.
$$

where $0<\beta<\alpha<1,{ }^{\mathrm{CF}} \mathrm{D}^{\alpha} x,{ }^{\mathrm{CF}} \mathrm{D}^{\beta} \mathrm{x}$ are the Caputo-Fabrizio fractional derivative defined in [14], by

$$
\begin{aligned}
& { }^{C F} D^{\alpha} x(t)=\frac{M(\alpha)}{1-\alpha} \int_{0}^{t} \exp \left(-\frac{\alpha}{1-\alpha}(t-s)\right) x^{\prime}(s) d s, \quad t \geqslant 0, \\
& { }^{C F} D^{\beta} x(t)=\frac{M(\beta)}{1-\beta} \int_{0}^{t} \exp \left(-\frac{\beta}{1-\beta}(t-s)\right) x^{\prime}(s) d s, \quad t \geqslant 0,
\end{aligned}
$$

\footnotetext{
${ }^{*}$ Corresponding author

Email addresses: zsqjk@163.com (Shuqin Zhang), huleimath@163.com (Lei Hu), kdssj@163.com (Sujing Sun)

doi: $10.22436 /$ jnsa.011.03.11
}

Received: 2017-10-14 Revised: 2017-12-17 Accepted: 2018-01-01 
where $M(\alpha), M(\beta)$ are normalization functions depending on $\alpha$ and $\beta$ such that $M(0)=M(1)=1$. Also, in [4] authors obtained the Laplace transform of the Caputo-Fabrizio fractional derivative

$$
L\left[{ }^{C F} D^{\alpha} f(t)\right](s)=\frac{M(\alpha)}{s+\alpha(1-s)}(s L[f(t)](s)-f(0)), s>0,
$$

where $L[f(t)]$ denotes the Laplace transform of function $f$.

In $[1,14]$, authors studied some properties of Caputo-Fabrizio fractional derivative defined in (1.2). In [22], authors consider the expression of solutions of some class of linear differential equations for the fractional derivative defined as following

$$
{ }^{\mathrm{CF}} \mathrm{D}_{*}^{\alpha} x(\mathrm{t})=\frac{(2-\alpha) \mathrm{M}_{1}(\alpha)}{2(1-\alpha)} \int_{0}^{\mathrm{t}} \exp \left(-\frac{\alpha}{1-\alpha}(\mathrm{t}-\mathrm{s})\right) x^{\prime}(\mathrm{s}) \mathrm{d} s, \mathrm{t} \geqslant 0,
$$

where $M_{1}(\alpha)$ is a normalization function depending on $\alpha$. Obviously, if let $M_{1}(\alpha)=\frac{2 M(\alpha)}{2-\alpha}$, here $M(\alpha)$ is the normalization function such that $M(0)=M(1)=1$, then fractional derivative defined in (1.3) is the Caputo-Fabrizio fractional derivative defined in (1.2).

In $[5,11]$, authors considered the existence of solutions of some differential and integro-differential equations involving with the Caputo-Fabrizio derivative. There are many works concerning with the applications and some partial differential equations of Caputo-Fabrizio derivative, for the details, please see $[2-4,12,13,15,17,21,23,24]$.

Fractional calculus (integrals and derivatives) are the generalizations of integer-order differential and integral operators. It is well known that the motivation for those works rises from both the development of the theory of fractional calculus itself and the applications of such constructions in various sciences such as physics, chemistry, aerodynamics, electro-dynamics of complex medium, polymer rheology, etc. For details, see $[6,18,19]$. There are many papers which discussed the existence of solutions to initial or boundary value problems for fractional differential equations denoted by the Rieamnn-Liouville fractional derivative or the Caputo fractional derivative, see $[7-10,16,20]$.

Motivated by these, in this paper, we explore the existence of the solution of the problem (1.1).

This paper is organized as follows. In Section 2, we study the expression of the solution of an initial value problem studied in [22] for linear differential equation for the fractional derivative defined in (1.3). In Section 3, using results obtained in Section 2, we consider the unique existence of solutions of an initial value problem for nonlinear differential equation denoted by the Caputo-Fabrizio fractional derivative.

\section{Linear equations}

In [22], authors obtained the following result.

Lemma 2.1 ([22]). Let $0<\alpha<1$. Then the unique solution of the following initial value problem

$$
\left\{\begin{array}{l}
{ }^{C F} D_{*}^{\alpha} f(t)=\sigma(t), t>0, \\
f(0)=f_{0} \in R
\end{array}\right.
$$

is given by

$$
f(t)=f_{0}+a_{\alpha}(\sigma(t)-\sigma(0))+b_{\alpha} I^{1} \sigma(t), t \geqslant 0,
$$

where $\mathrm{I}^{1}$ denotes a primitive of $\sigma$ and

$$
a_{\alpha}=\frac{2(1-\alpha)}{(2-\alpha) M_{1}(\alpha)}, \quad b_{\alpha}=\frac{2 \alpha}{(2-\alpha) M_{1}(\alpha)}
$$

According to the definition of ${ }^{\mathrm{CF}} \mathrm{D}_{*}^{\alpha}$ defined in (1.3), we find out that Lemma 2.1 is incorrect, except that $\sigma(0)=0$. With careful deduction, we obtain the following result. 
Theorem 2.2. Let $0<\alpha<1, \sigma:[0,+\infty) \rightarrow \mathrm{R}$ be a continuous differential function, then the solution of the following initial value problem

$$
\left\{\begin{array}{l}
{ }^{C F} D_{*}^{\alpha} f(t)=\sigma(t)-\sigma(0) e^{-\frac{\alpha}{1-\alpha} t}, t>0 \\
f(0)=f_{0} \in R
\end{array}\right.
$$

is given by

$$
f(t)=f_{0}+a_{\alpha}(\sigma(t)-\sigma(0))+b_{\alpha} I^{1} \sigma(t), \quad t \geqslant 0,
$$

where $\mathrm{I}^{1}$ denotes a primitive of $\sigma$ and

$$
a_{\alpha}=\frac{2(1-\alpha)}{(2-\alpha) M_{1}(\alpha)}, \quad b_{\alpha}=\frac{2 \alpha}{(2-\alpha) M_{1}(\alpha)} .
$$

Proof. We verify that function defined in (2.2) is the solution of the problem (2.1). By (2.2), we get

$$
f^{\prime}(t)=a_{\alpha} \sigma^{\prime}(t)+b_{\alpha} \sigma(t), t \geqslant 0 .
$$

Hence, multiplied by function $\exp \left(\frac{\alpha}{1-\alpha} t\right)$ on both sides of (2.3), for $t \geqslant 0$, we have

$$
\exp \left(\frac{\alpha}{1-\alpha} t\right) f^{\prime}(t)=\exp \left(\frac{\alpha}{1-\alpha} t\right)\left(a_{\alpha} \sigma^{\prime}(t)+b_{\alpha} \sigma(t)\right)=a_{\alpha}\left(\exp \left(\frac{\alpha}{1-\alpha} t\right) \sigma(t)\right)^{\prime} .
$$

Integrating from 0 to $t$ on both sides of (2.4), for $t \geqslant 0$, we have

$$
\int_{0}^{t} \exp \left(\frac{\alpha}{1-\alpha} s\right) f^{\prime}(s) d s=\frac{2(1-\alpha)}{(2-\alpha) M_{1}(\alpha)}\left(\exp \left(\frac{\alpha}{1-\alpha} t\right) \sigma(t)-\sigma(0)\right) .
$$

From (2.5), for $t \geqslant 0$, we get

$$
\frac{(2-\alpha) M_{1}(\alpha)}{2(1-\alpha)} \int_{0}^{t} \exp \left(-\frac{\alpha}{1-\alpha}(t-s)\right) f^{\prime}(s) d s=\sigma(t)-\sigma(0) \exp \left(-\frac{\alpha}{1-\alpha} t\right) .
$$

As well, from (2.2), we know that $f(0)=f_{0}$. With (2.6) combined, we get that $f(t)$ is the solution of the problem (2.1). Thus, we complete the proof.

We also obtain the following result.

Theorem 2.3. Let $0<\alpha<1, \sigma:[0,+\infty) \rightarrow \mathrm{R}$ be a continuous differential function, then the solution of the following initial value problem

$$
\left\{\begin{array}{l}
\mathrm{CF}_{*}^{\alpha} f(t)=\sigma(t)-\sigma(0), t>0 \\
f(0)=f_{0} \in R
\end{array}\right.
$$

is given by

$$
f(t)=f_{0}+a_{\alpha}(\sigma(t)-\sigma(0))+b_{\alpha} I^{1} \sigma(t)-b_{\alpha} \sigma(0) t, \quad t \geqslant 0,
$$

where $\mathrm{I}^{1}$ denotes a primitive of $\sigma$ and

$$
a_{\alpha}=\frac{2(1-\alpha)}{(2-\alpha) M_{1}(\alpha)}, \quad b_{\alpha}=\frac{2 \alpha}{(2-\alpha) M_{1}(\alpha)} .
$$

Proof. Similar to Theorem 2.2, here, we test that function defined in (2.8) is the solution of the problem (2.7). By (2.8), we get

$$
f^{\prime}(t)=a_{\alpha} \sigma^{\prime}(t)+b_{\alpha} \sigma(t)-b_{\alpha} \sigma(0), t \geqslant 0 .
$$

Multiplying by function $\exp \left(\frac{\alpha}{1-\alpha} t\right)$ on both sides of (2.9), we get 


$$
\begin{aligned}
\exp \left(\frac{\alpha}{1-\alpha} t\right) f^{\prime}(t) & =\exp \left(\frac{\alpha}{1-\alpha} t\right)\left(a_{\alpha} \sigma^{\prime}(t)+b_{\alpha} \sigma(t)\right)-b_{\alpha} \sigma(0) \exp \left(\frac{\alpha}{1-\alpha} t\right) \\
& =\frac{2(1-\alpha)}{(2-\alpha) M_{1}(\alpha)}\left(\exp \left(\frac{\alpha}{1-\alpha} t\right) \sigma(t)\right)^{\prime}-\exp \left(\frac{\alpha}{1-\alpha} t\right) \frac{2 \alpha}{(2-\alpha) M_{1}(\alpha)} \sigma(0) .
\end{aligned}
$$

Integrating now from 0 to $t$ on both sides of the equality above, we have

$$
\begin{aligned}
\int_{0}^{t} \exp \left(\frac{\alpha}{1-\alpha} s\right) f^{\prime}(s) d s & =\frac{2(1-\alpha)}{(2-\alpha) M_{1}(\alpha)}\left(\exp \left(\frac{\alpha}{1-\alpha} t\right) \sigma(t)-\sigma(0)\right)-\frac{2(1-\alpha) \sigma(0)}{(2-\alpha) M_{1}(\alpha)}\left(\exp \left(\frac{\alpha}{1-\alpha} t\right)-1\right) \\
& =\frac{2(1-\alpha)}{(2-\alpha) M_{1}(\alpha)} \exp \left(\frac{\alpha}{1-\alpha} t\right) \sigma(t)-\frac{2(1-\alpha)}{(2-\alpha) M_{1}(\alpha)} \exp \left(\frac{\alpha}{1-\alpha} t\right) \sigma(0) .
\end{aligned}
$$

According to the equality above, we get

$$
\frac{(2-\alpha) M_{1}(\alpha)}{2(1-\alpha)} \int_{0}^{t} \exp \left(-\frac{\alpha}{1-\alpha}(t-s)\right) f^{\prime}(s) d s=\sigma(t)-\sigma(0), t \geqslant 0 .
$$

By (2.8), we know that $f(0)=f_{0}$. Hence, $f(t)$ defined in (2.8) is the solution of the problem (2.7).

From Theorem 2.3, for the problem (1.1), we have the following result.

Lemma 2.4. Let $0<\alpha<1, \mathrm{~h}:[0,+\infty) \rightarrow \mathrm{R}$ be a continuous differential function, then the solution of the following initial value problem

$$
\left\{\begin{array}{l}
{ }^{C F} D^{\alpha} f(t)=h(t)-h(0), t>0 \\
f(0)=f_{0} \in R
\end{array}\right.
$$

is given by

$$
f(t)=f_{0}+a_{\alpha}(h(t)-h(0))+b_{\alpha} I^{1} h(t)-b_{\alpha} h(0) t, t \geqslant 0,
$$

where $\mathrm{I}^{1}$ denotes a primitive of $\sigma$ and

$$
a_{\alpha}=\frac{1-\alpha}{M(\alpha)}, \quad b_{\alpha}=\frac{\alpha}{M(\alpha)}
$$

Proof. From (1.2), (1.3), and Theorem 2.3, let $M_{1}(\alpha)=\frac{2 M(\alpha)}{2-\alpha}$, here $M(\alpha)$ is the normalization function such that $M(0)=M(1)=1$, then we could get that the function in (2.11) is the solution of the problem (2.10).

\section{Nonlinear equations}

In this section, we consider the existence of solution of the problem (1.1). Our main result is the following.

Theorem 3.1. Let $0<\beta<\alpha<1, g:[0,+\infty) \times R \times R \rightarrow R$ be a continuous differentiable function, there exist $\mathrm{L}_{1}>0, \mathrm{~L}_{2}>0$ satisfying $\mathrm{L}_{1}<\frac{\mathrm{M}(\alpha)}{4}, \mathrm{~L}_{2}<\frac{\mathrm{M}(\alpha)}{4(\mathrm{~A}+\mathrm{B})}$ and $\lambda>2$, such that for all $\mathrm{t} \in[0,+\infty), x, y \in \mathrm{R}$,

$$
\left|g\left(t,\left(1+t^{\lambda}\right) x,\left(1+t^{\lambda}\right)^{C F} D^{\beta} x\right)-g\left(t,\left(1+t^{\lambda}\right) y,\left(1+t^{\lambda}\right)^{C F} D^{\beta} y\right)\right| \leqslant L_{1}|x-y|+\left.L_{2}\right|^{C F} D^{\beta} x-{ }^{C F} D^{\beta} y \mid
$$

holds, and that

$$
\lim _{t \rightarrow+\infty} \frac{|g(t, 0,0)|+\int_{0}^{t}|g(s, 0,0)| d s}{1+t^{\lambda}}=0
$$

where

$$
A=\frac{2 M(\beta)}{1-\beta}, B=\frac{\beta M(\beta)}{(1-\beta)^{2}} .
$$

Then the problem (1.1) has one unique solution. 
Proof. Let

$$
E=\left\{x(t) \mid x(t) \in C[0,+\infty), \sup _{t \geqslant 0} \frac{x(t)}{1+t^{\lambda}}<\infty\right\}
$$

with the norm

$$
\|x\|_{E}=\sup _{t \geqslant 0} \frac{|x(t)|}{1+t^{\lambda}}
$$

then, by the same arguments as in [2, Lemma 2.2], we could know that $\left(E,\|\cdot\|_{E}\right)$ is a Banach space, here we omit this proof.

For $x \in E$, we have

$$
|x(0)|=\left.\frac{|x(t)|}{1+t^{\lambda}}\right|_{t=0} \leqslant \sup _{t \geqslant 0} \frac{|x(t)|}{1+t^{\lambda}}=\|x\|_{E} .
$$

From Lemma 2.4, in order to obtain the existence result of solution of the problem (1.1), it is sufficient to consider the existence of fixed point $\mathrm{T}: \mathrm{E} \rightarrow \mathrm{E}$, defined as

$$
\begin{aligned}
\mathrm{T} x(t)= & x_{0}+\frac{1-\alpha}{M(\alpha)}\left(g\left(t, x(t),{ }^{C F} D^{\beta} x(t)\right)-g\left(0, x(0),{ }^{C F} D^{\beta} x(0)\right)\right) \\
& +\frac{\alpha}{M(\alpha)} \int_{0}^{t} g\left(s, x(s),{ }^{C F} D^{\beta} x(s)\right) d s-\frac{\alpha g\left(0, x(0),{ }^{C F} D^{\beta} x(0)\right)}{M(\alpha)} t, 0 \leqslant t<+\infty .
\end{aligned}
$$

Operator $\mathrm{Tx}(\mathrm{t}) \in \mathrm{C}[0,+\infty)$ for $x \in \mathrm{E}$ is obtained through the continuity assumption of function $\mathrm{g}$.

By the definition of the Caputo-Fabrizio fractional derivative, we know that

$$
\begin{aligned}
{ }^{C F} D^{\beta} x(t) & =\frac{M(\beta)}{1-\beta} \int_{0}^{t} \exp \left(-\frac{\beta}{1-\beta}(t-s)\right) x^{\prime}(s) d s \\
& =\frac{M(\beta)}{1-\beta} x(t)-\frac{M(\beta)}{1-\beta} \exp \left(-\frac{\beta}{1-\beta} t\right) x(0)-\frac{\beta M(\beta)}{(1-\beta)^{2}} \int_{0}^{t} \exp \left(-\frac{\beta}{1-\beta}(t-s)\right) x(s) d s, \quad 0 \leqslant t<+\infty .
\end{aligned}
$$

For $x \in E$, from the equality above, we have

$$
\begin{aligned}
\left|\frac{{ }^{C F} D^{\beta} x(t)}{1+t^{\lambda}}\right| & =\left|\frac{M(\beta)}{1-\beta} \frac{x(t)}{1+t^{\lambda}}-\frac{M(\beta) x(0)}{(1-\beta)\left(1+t^{\lambda}\right)} \exp \left(-\frac{\beta}{1-\beta} t\right)-\frac{\beta M(\beta)}{(1-\beta)^{2}\left(1+t^{\lambda}\right)} \int_{0}^{t} \exp \left(-\frac{\beta}{1-\beta}(t-s)\right) x(s) d s\right| \\
& \leqslant \frac{2 M(\beta)}{1-\beta}\|x\|_{E}+\frac{\beta M(\beta)\|x\|_{E}}{(1-\beta)^{2}\left(1+t^{\lambda}\right)} \int_{0}^{t}\left(1+s^{\lambda}\right) d s \\
& \leqslant \frac{2 M(\beta)}{1-\beta}\|x\|_{E}+\frac{\beta M(\beta)\|x\|_{E}}{(1-\beta)^{2}\left(1+t^{\lambda}\right)} \int_{0}^{t}\left(1+t^{\lambda}\right) d s \\
& =\left(\frac{2 M(\beta)}{1-\beta}+\frac{\beta M(\beta) t}{(1-\beta)^{2}}\right)\|x\|_{E}=(A+B t)\|x\|_{E}, \quad 0 \leqslant t<+\infty,
\end{aligned}
$$

where $A, B$ are the constants defined in (3.3).

For $x \in E$, by (3.1) and (3.4), we have

$$
\begin{aligned}
\left|g\left(t, x(t),{ }^{C F} D^{\beta} x(t)\right)\right| & =\left|g\left(t, x(t),{ }^{C F} D^{\beta} x(t)\right)-g(t, 0,0)+g(t, 0,0)\right| \\
& \leqslant L_{1}\left|\frac{x(t)}{1+t^{\lambda}}\right|+L_{2}\left|\frac{{ }^{C F} D^{\beta} x(t)}{1+t^{\lambda}}\right|+|g(t, 0,0)| \\
& \leqslant\left(L_{1}+L_{2}(A+B t)\right)\|x\|_{E}+|g(t, 0,0)| \\
& =\left(L_{1}+L_{2} A+L_{2} B t\right)\|x\|_{E}+|g(t, 0,0)|, \\
\left|g\left(0, x(0),{ }^{C F} D^{\beta} x(0)\right)\right| & =\left|g\left(0, x(0),{ }^{C F} D^{\beta} x(0)\right)-g(0,0,0)+g(0,0,0)\right| \\
& \leqslant L_{1}|x(0)|+L_{2}\left|{ }^{C F} D^{\beta} x(0)\right|+|g(0,0,0)|
\end{aligned}
$$




$$
\begin{aligned}
\leqslant & \left(L_{1}+L_{2}(A+B t)\right)\|x\|_{E}+|g(0,0,0)| \\
= & \left(L_{1}+L_{2} A+L_{2} B t\right)\|x\|_{E}+|g(0,0,0)| \\
\left|\frac{T x(t)}{1+t^{\lambda}}\right| \leqslant & \frac{1-\alpha}{M(\alpha)\left(1+t^{\lambda}\right)}\left|g\left(t, x(t),{ }^{C F} D^{\beta} x(t)\right)-g\left(0, x(0),{ }^{C F} D^{\beta} x(0)\right)\right| \\
& +\frac{\alpha}{M(\alpha)\left(1+t^{\lambda}\right)} \int_{0}^{t}\left|g\left(s, x(s), C F D^{\beta} x(s)\right)\right| d s \\
& +\frac{\alpha}{M(\alpha)}\left|g\left(0, x(0),{ }^{C F} D^{\beta} x(0)\right)\right| \frac{t}{1+t^{\lambda}}+\frac{\left|x_{0}\right|}{1+t^{\lambda}} \\
\leqslant & \frac{\left|x_{0}\right|}{1+t^{\lambda}}+\frac{(1-\alpha)\left(\left(2 L_{1}+2 L_{2} A+2 L_{2} B t\right)\|x\|_{E}+|g(t, 0,0)|+|g(0,0,0)|\right)}{M(\alpha)\left(1+t^{\lambda}\right)} \\
& +\frac{\left.\alpha\left(\left(L_{1}+L_{2} A\right) t+\frac{L_{2} B t^{2}}{2}\right)\|x\|_{E}+\int_{0}^{t}|g(s, 0,0)| d s\right)}{M(\alpha)\left(1+t^{\lambda}\right)} \\
& +\frac{\left.\alpha\left(\left(L_{1}+L_{2} A\right) t+L_{2} B t^{2}\right)\|x\|_{E}+|g(0,0,0)| t\right)}{M(\alpha)\left(1+t^{\lambda}\right)} \\
\leqslant & \frac{\left|x_{0}\right|}{1+t^{\lambda}}+\frac{(1-\alpha)\left(\left(2 L_{1}+2 L_{2} A+2 L_{2} B t\right)\|x\|_{E}+|g(t, 0,0)|+|g(0,0,0)|\right)}{M(\alpha)\left(1+t^{\lambda}\right)} \\
& +\frac{\left.2 \alpha\left(\left(L_{1}+L_{2} A\right) t+L_{2} B t^{2}\right)\|x\|_{E}+\alpha \int_{0}^{t}|g(s, 0,0)| d s+\alpha|g(0,0,0)| t\right)}{M(\alpha)\left(1+t^{\lambda}\right)} \\
\leqslant & \frac{\left(2(1-\alpha)\left(L_{1}+L_{2} A\right)+\left(2 L_{2} B(1-\alpha)+2 \alpha\left(L_{1}+L_{2} A\right)\right) t+2 \alpha L_{2} B t^{2}\right)\|x\|_{E}}{M(\alpha)\left(1+t^{\lambda}\right)} \\
& +\frac{\left|x_{0}\right|}{1+t^{\lambda}}+\frac{1}{M(\alpha)} \max \{\alpha, 1-\alpha\} \frac{\int_{0}^{t}|g(s, 0,0)| d s+|g(t, 0,0)|}{1+t^{\lambda}} \\
& \frac{(1-\alpha)|g(0,0,0)|+\alpha|g(0,0,0)| t}{M(\alpha)\left(1+t^{\lambda}\right)} \\
&
\end{aligned}
$$

by (3.2), we get that $\lim _{t \rightarrow+\infty} \frac{T x(t)}{1+t^{\lambda}}=0$. Hence, we obtain that $T: E \rightarrow E$ is well defined.

Now, we verify that $T: E \rightarrow E$ is a contraction operator.

For $x, y \in E$, from the previous arguments, we obtain

$$
\left|\frac{{ }^{C F} D^{\beta} x(t)}{1+t^{\lambda}}-\frac{{ }^{C F} D^{\beta} y(t)}{1+t^{\lambda}}\right| \leqslant(A+B t)\|x-y\|_{E}, \quad 0 \leqslant t<+\infty
$$

For $x, y \in E$, by (3.1) and (3.5), we get

$$
\begin{aligned}
\left|\frac{T x(t)}{1+t^{\lambda}}-\frac{T y(t)}{1+t^{\lambda}}\right| \leqslant & \frac{1-\alpha}{M(\alpha)\left(1+t^{\lambda}\right)}\left[L_{1}\left|\frac{x(t)}{1+t^{\lambda}}-\frac{y(t)}{1+t^{\lambda}}\right|+L_{1}|x(0)-y(0)|\right. \\
& \left.+L_{2}\left|\frac{{ }^{C F} D^{\beta} x(t)}{1+t^{\lambda}}-\frac{{ }^{C F} D^{\beta} y(t)}{1+t^{\lambda}}\right|+\left.L_{2}\right|^{C F} D^{\beta} x(0)-{ }^{C F} D^{\beta} y(0) \mid\right] \\
& +\frac{\alpha}{M(\alpha)\left(1+t^{\lambda}\right)} \int_{0}^{t} L_{1}\left|\frac{x(s)}{1+t^{\lambda}}-\frac{y(s)}{1+t^{\lambda}}\right|+L_{2}\left|\frac{{ }^{C F} D^{\beta} x(s)}{1+t^{\lambda}}-\frac{{ }^{C F} D^{\beta} y(s)}{1+t^{\lambda}}\right| d s \\
& +\frac{\alpha t}{M(\alpha)\left(1+t^{\lambda}\right)}\left(L_{1}|x(0)-y(0)|+L_{2}\left|{ }^{C F} D^{\beta} x(0)-{ }^{C F} D^{\beta} y(0)\right|\right) \\
\leqslant & {\left[\frac{2(1-\alpha) L_{1}}{M(\alpha)\left(1+t^{\lambda}\right)}+\frac{2(1-\alpha) L_{2}(A+B t)}{M(\alpha)\left(1+t^{\lambda}\right)}\right]\|x-y\|_{E} } \\
& +\frac{\alpha}{M(\alpha)\left(1+t^{\lambda}\right)} \int_{0}^{t}\left(L_{1}+L_{2} A+L_{2} B s\right) d s\|x-y\|_{E}
\end{aligned}
$$




$$
\begin{aligned}
& +\frac{\alpha t}{M(\alpha)\left(1+t^{\lambda}\right)}\left(L_{1}+L_{2} A+L_{2} B t\right)\|x-y\|_{E} \\
\leqslant & {\left[\frac{2(1-\alpha) L_{1}}{M(\alpha)}+\frac{2(1-\alpha) L_{2}(A+B)}{M(\alpha)}\right]\|x-y\|_{E} } \\
& +\left[\frac{L_{1} \alpha}{M(\alpha)}+\frac{L_{2} \alpha(A+B)}{M(\alpha)}\right]\|x-y\|_{E}+\left[\frac{L_{1} \alpha}{M(\alpha)}+\frac{L_{2} \alpha(A+B)}{M(\alpha)}\right]\|x-y\|_{E} \\
= & {\left[\frac{2 L_{1}}{M(\alpha)}+\frac{2 L_{2}(A+B)}{M(\alpha)}\right]\|x-y\|_{E} . }
\end{aligned}
$$

It follows from $L_{1}<\frac{M(\alpha)}{4}$ and $L_{2}<\frac{M(\alpha)}{4(A+B)}$ that $\frac{2 L_{1}}{M(\alpha)}+\frac{2 L_{2}(A+B)}{M(\alpha)}<1$, which implies that $T: E \rightarrow E$ is a contraction operator. Then, the Banach contraction principle assures that operator $T$ has a unique fixed point $x \in E$, which means that there exists a unique solution $x \in E$ for problem (1.1). Thus, we complete this proof.

\section{An example}

Example 4.1. Consider the following initial value problem of differential equation involving the CaputoFabrizio fractional derivative

$$
\left\{\begin{array}{l}
{ }^{C F} D^{\frac{3}{4}} x(t)=\frac{t^{\frac{1}{2}}}{2}+\frac{5 M\left(\frac{3}{4}\right) x^{2} \sin t}{68\left(1+t^{2}\right)^{2}\left(1+x^{2}\right)}+\frac{5 M\left(\frac{3}{4}\right)\left({ }^{C F} D^{\frac{1}{2}} x\right)^{2} \sin t^{2}}{290\left(1+t^{2}\right)^{2} M\left(\frac{1}{2}\right)\left(1+\left({ }^{C F} D^{\frac{1}{2}} x\right)^{2}\right)}, 0 \leqslant t<\infty, \\
x(0)=0 .
\end{array}\right.
$$

Let $g\left(t, x,{ }^{C F} D^{\frac{1}{2}} x\right)=\frac{t^{\frac{1}{2}}}{2}+\frac{5 M\left(\frac{3}{4}\right) x^{2} \sin t}{68\left(1+t^{2}\right)^{2}\left(1+x^{2}\right)}+\frac{5 M\left(\frac{3}{4}\right)\left({ }^{C F} D^{\frac{1}{2}} x\right)^{2} \sin t^{2}}{290\left(1+t^{2}\right)^{2} M\left(\frac{1}{2}\right)\left(1+\left({ }^{C F} D^{\frac{1}{2}} x\right)^{2}\right)}$. Obviously, $g:[0,+\infty) \times R \times R \rightarrow R$ is a continuous differentiable function, $g\left(0, x(0),{ }^{\mathrm{CF}} \mathrm{D}^{\frac{1}{2}} \mathrm{x}(0)\right)=0$. Let

$$
\begin{aligned}
G(t, x, y) & =g\left(t,\left(1+t^{2}\right) x,\left(1+t^{2}\right) y\right) \\
& =\frac{t^{\frac{1}{2}}}{2}+\frac{5 M\left(\frac{3}{4}\right) x^{2} \sin t}{68 x^{2}}+\frac{5 M\left(\frac{3}{4}\right)\left(1+t^{2}\right)^{2} y^{2} \sin t^{2}}{290 M\left(\frac{1}{2}\right)\left(1+\left(1+t^{2}\right)^{2} y^{2}\right)}, \quad 0 \leqslant t<\infty, x, y \in R .
\end{aligned}
$$

For all $0 \leqslant t<\infty, x, y \in R$, with simple calculation, we obtain

$$
G_{x}(t, x, y)=\frac{5 M\left(\frac{3}{4}\right) \sin t}{34} \frac{\left(1+t^{2}\right)^{2} x}{\left(1+\left(1+t^{2}\right)^{2} x^{2}\right)^{2}} \quad, \quad G_{y}(t, x, y)=\frac{5 M\left(\frac{3}{4}\right) \sin t^{2}}{145 M\left(\frac{1}{2}\right)} \frac{\left(1+t^{2}\right)^{2} y}{\left(1+\left(1+t^{2}\right)^{2} y^{2}\right)^{2}} .
$$

Obviously, for all $0 \leqslant t<\infty, x, y \in R$, we have

$$
\left|G_{x}(t, x, y)\right| \leqslant \frac{5 M\left(\frac{3}{4}\right)}{34}, \quad\left|G_{y}(t, x, y)\right| \leqslant \frac{5 M\left(\frac{3}{4}\right)}{145 M\left(\frac{1}{2}\right)} .
$$

Hence, for all $t \in[0,+\infty), x \in R$, it holds

$$
\begin{aligned}
& \left|g\left(t,\left(1+t^{2}\right) x,\left(1+t^{2}\right){ }^{C F} D^{\frac{1}{2}} x\right)-g\left(t,\left(1+t^{2}\right) y,\left(1+t^{2}\right)^{C F} D^{\frac{1}{2}} y\right)\right| \\
& \quad=\left|G\left(t, x,{ }^{C F} D^{\frac{1}{2}} x\right)-G\left(t, y,{ }^{C F} D^{\frac{1}{2}} y\right)\right| \leqslant \frac{5 M\left(\frac{3}{4}\right)}{34}|x-y|+\frac{5 M\left(\frac{3}{4}\right)}{145 M\left(\frac{1}{2}\right)}\left|{ }^{C F} D^{\frac{1}{2}} x-{ }^{C F} D^{\frac{1}{2}} y\right|,
\end{aligned}
$$

which implies that $g$ satisfies (3.1) of Theorem 3.1, with $L_{1}=\frac{5 M\left(\frac{3}{4}\right)}{34}, L_{2}=\frac{5 M\left(\frac{3}{4}\right)}{145 M\left(\frac{1}{2}\right)}, \lambda=2$. Meanwhile, by calculating, we obtain

$$
A+B=\frac{2 M(\beta)}{1-\beta}+\frac{\beta M(\beta)}{(1-\beta)^{2}}=4 M\left(\frac{1}{2}\right)+2 M\left(\frac{1}{2}\right)=6 M\left(\frac{1}{2}\right) .
$$


Hence, we have

$$
\mathrm{L}_{1}=\frac{5 \mathrm{M}\left(\frac{3}{4}\right)}{34}<\frac{\mathrm{M}\left(\frac{3}{4}\right)}{4}=\frac{M(\alpha)}{4}, \quad \mathrm{~L}_{2}=\frac{5 \mathrm{M}\left(\frac{3}{4}\right)}{145 \mathrm{M}\left(\frac{1}{2}\right)}<\frac{\mathrm{M}\left(\frac{3}{4}\right)}{24 \mathrm{M}\left(\frac{1}{2}\right)}=\frac{\mathrm{M}(\alpha)}{4(\mathrm{~A}+\mathrm{B})} .
$$

And that

$$
\lim _{t \rightarrow+\infty} \frac{|g(t, 0,0)|+\int_{0}^{t}|g(s, 0,0)| d s}{1+t^{2}}=\lim _{t \rightarrow+\infty} \frac{t^{\frac{1}{2}}+\int_{0}^{t} s^{\frac{1}{2}} d s}{2\left(1+t^{2}\right)}=0 .
$$

Then, we see that all assumption conditions in Theorem 3.1 are satisfied. Hence, by Theorem 3.1, the problem (4.1) have a unique solution $x \in E$.

\section{Acknowledgment}

The authors are thankful to the referees for their careful reading of the manuscript and insightful comments. This research is supported by the Natural Science Foundation of China $(11371364,11671181$, 11571207).

\section{References}

[1] T. Abdeljawad, D. Baleanu, On fractional derivatives with exponential kernel and their discrete versions, Rep. Math. Phys., 80 (2017), 11-27. 1

[2] M. Abdulhameed, D. Vieru, R. Roslan, Modeling electro-magneto-hydrodynamic thermo-fluidic transpot of biofluids with new trend of fractional derivative without singular kernel, Phys. A, 484 (2017), 233-252. 1, 3.1

[3] N. Al-Salti, E. Karimov, K. Sadarangani, On a differential Equation with Caputo-Fabrizio fractional derivative of order $1<\beta \leqslant 2$ and applications to Mass-Spring-Damper system, Progr. Fract. Differ. Appl., 2 (2016), 257-263.

[4] A. Atangana, On the new fractional derivative and application to nonlinear Fisher's reaction-diffusion equation, Appl. Math. Comput., 273 (2016), 948-956. 1, 1

[5] S. Aydogan, D. Baleanu, A. Mousalou, S. Rezapour, On approximate solutions for two higher-order Caputo-Fabrizio fractional integro-differential equtions, Adv. Difference Equ., 2017 (2017), 11 pages. 1

[6] R. L. Bagley, P. J. Torvik, On the fractional calculus model of viscoelastic behavior, J. Rheol., 30 (1986), 133-155. 1

[7] Z. Bai, X. Dong, C. Yin, Existence results for impulsive nonlinear fractional differential equation with mixed boundary conditions, Bound. Value Probl., 2016 (2016), 11 pages. 1

[8] Z. Bai, T. Qiu, Existence of positive solution for singular fractional differential equation, Appl. Math. Comput., 215 (2009), 2761-2767.

[9] Z. Bai, Y. Zhang, The existence of solutions for a fractional multi-point boundary value problem, Comput. Math. Appl., 60 (2010), 2364-2372.

[10] Z. Bai, S. Zhang, S. Sun, C. Yin, Monotone iterative method for a class of fractional differential equations, Electron. J. Differential Equations, 2016 (2016), 8 pages. 1

[11] D. Baleanu, A. Mousalou, S. Rezapour, On the existence of solutions for some infinite coefficient-symmetric CaputoFabrizio fractional integro-differential equations, Bound. Value Probl., 2017 (2017), 9 pages. 1

[12] D. Baleanu, A. Mousalou, S. Rezapour, A new method for investigationg approximate solutions of some fractional integrodifferential equations involving the Caputo-Fabrizio derivative, Adv. Difference Equ., 2017 (2017), 12 pages. 1

[13] A. R. Butt, M. Abdullah, N. Raza, M. A. Imran, Influence of non-integer order parameter and Hartmann number on the heat and mass transfer flow of a Jeffery fluid over an oscillationg vertical plate via Caputo-Fabrizio tiem fractional derivatives, Eur. Phys. J. plus, 2017 (2017), 16 pages. 1

[14] M. Caputo, M. Fabrizio, A New Definition of Fractional Derivative without Singular Kernel, Progr. Fract. Differ. Appl., 1 (2015), 1-13. 1, 1

[15] M. Caputo, M. Fabrizio, Applications of New Time and Spatial Fractional Derivatives with Exponential Kernels, Progr. Fract. Differ. Appl., 2 (2016), 1-11. 1

[16] Y. Cui, Uniqueness of solution for boundary value problems for fractional differential equations, Appl. Math. Lett., 51 (2016), 48-54. 1

[17] J. F. Gómez-Aguilar, Space-time fractional diffusion equation using a derivative with nonsingular and regular kernel, Phys. A, 465 (2017), 562-572. 1

[18] R. Hilfer, Applications of Fractional calculus in Physics, World Scientific, Singapore, (2000). 1

[19] A. A. Kilbas, H. M. Srivastava, J. J. Trujillo, Theory and Applications of Fractional Differential Equations, Elsevier Science B.V., Amsterdam, (2006). 1

[20] C. Kou, H. Zhou, Y. Yan, Existence of solutions of initial value problems for nonlinear fractional differential equations on the half-axis, Nonlinear Anal., 74 (2011), 5975-5986. 1 
[21] D. Kumar, J. Singh, M. Al Qurashi, D. Baleanu, Analysis of logistic equation pertaining to a new fractional derivative with non-singular kernel, Adv. Mech. Eng., 2017 (2017), 8 pages. 1

[22] J. Losada, J. J. Nieto, Properties of a New Fractional Derivative without Singular Kernel, Progr. Fract. Differ. Appl., 1 (2015), 87-92. 1, 1, 2, 2.1

[23] I. A. Mirza, D. Vieru, Fundamental solutions to advection-diffusion equation with time-fractional Caputo-Fabrizio derivative, Comput. Math. Appl., 73 (2017), 1-10. 1

[24] J. Singh, D. Kumar, Z. Hammouch, A. Atangana, A fractional epidemiological model for computer viruses pertaining to a new fractional derivative, Appl. Math. Comput., 316 (2018), 504-515. 1 